\title{
Combined CT angiography of the aorta and craniocervical artery: a new imaging protocol for assessment of acute type A aortic dissection
}

\author{
Didi Wen ${ }^{1}$, Hongliang Zhao ${ }^{1}$, Weixun Duan ${ }^{2}$, Rui An ${ }^{1}$, Jian Li $^{1}$, Minwen Zheng ${ }^{1}$ \\ ${ }^{1}$ Department of Radiology, ${ }^{2}$ Department of Cardiovascular Surgery, Xijing Hospital, Fourth Military Medical University, Xi'an 710032 , China \\ Contributions: (I) Conception and design: M Zheng; (II) Administrative support: D Wen, R An; (III) Provision of study materials or patients: W Duan; \\ (IV) Collection and assembly of data: H Zhao, J Li; (V) Data analysis and interpretation: H Zhao, J Li; (VI) Manuscript writing: All authors; (VII) \\ Final approval of manuscript: All authors. \\ Correspondence to: Minwen Zheng, MD. Department of Radiology, Xijing Hospital, Fourth Military Medical University, 127\# West Changle Road, \\ Xi'an 710032, China. Email: zhengmw2007@163.com.
}

\begin{abstract}
Background: To investigate the feasibility of combined computed tomography angiography (CTA) of the aorta and craniocervical artery in acute type A aortic dissection (ATAAD) and the value of incremental craniocervical information.

Methods: Combined CTA of head, neck and aorta was performed in patients with suspected aortic dissection and 243 ATAAD patients were analyzed. The image quality and radiation dose were assessed. Valuable craniocervical CTA findings were determined for further surgical analysis.

Results: The mean dose-length product (DLP) was $314.11 \pm 29.31 \mathrm{mGy} . \mathrm{cm}$. The image quality of craniocervical arteries were $100 \%$ diagnostic. Intimal flap involving carotid arteries was detected in $47 \%$ of patients, and significant stenosis of true lumen was observed in $60 \%$ of common carotid arteries (CCAs). Hypodensity and hypoplasia/occlusion of carotid arteries were also detected and apprised surgeons. The tortuosity of carotid artery was found in $90.9 \%$ of patients. Bilateral antegrade selective cerebral perfusion (ASCP) was performed in $21.1 \%$ of aortic arch surgery based on 7 hemodynamic variation types of the circle of Willis (CW) determined by cranial CTA information.

Conclusions: A combined CTA of head, neck and aorta is feasible with low radiation dose and diagnostic image quality. Incremental information on craniocervical pathology and anatomy may be useful for surgery repair of ATAAD.
\end{abstract}

Keywords: Computed tomography angiography (CTA); aortic dissection; circle of Willis (CW); cerebral protection; aortic surgery

Submitted Jun 20, 2017. Accepted for publication Sep 01, 2017.

doi: $10.21037 /$ jtd.2017.09.28

View this article at: http://dx.doi.org/10.21037/jtd.2017.09.28

\section{Introduction}

According to the International Registry of Acute Aortic Dissection (IRAD) data, computed tomography angiography (CTA) may be presently the imaging modality of first choice on suspicion of aortic dissection (1). However, the scan range of conventional aortic CTA for assessment of aortic dissection is commonly from the thoracic inlet to the pelvis $(2,3)$, covering the proximal supraaortic branches to iliac artery. Hence, conventional aortic CTA fails to visualize the anatomy and pathology of cerebral and carotid arteries, which are important for the surgeon to plan pre- and intraoperative procedures in aortic arch surgery. The selection of reliable cerebral protection method and precise surgical procedures may influence the postoperative outcome.

Acute type A aortic dissection (ATAAD) is a life-threatening vascular emergency, mandating immediate surgical repair (4), 
and the complex operative procedures are associated with a relatively high mortality and neurologic complications (5). Cases requiring arch repair like total arch replacement, unilateral antegrade selective cerebral perfusion (ASCP) is usually the cerebral protection method of choice to prevent neurologic complications from occurring in ATAAD patients (6). Recent studies have been shown that the occurrence of temporary neurologic dysfunction (TND) is closely associated with the method of brain protection and the duration of deep hypothermic circulatory arrest (HCA) $(5,7)$.

During unilateral ASCP, the brain receives blood only through right common carotid artery (CCA) and right vertebral artery (VA). It is insufficient to maintain adequate perfusion in the left hemisphere when variations existing in both the anterior and posterior portion of the circle of Willis (CW). Hence, latent hypoperfusion of the left cerebral hemisphere is the major concern of unilateral ASCP due to the high incidence of the variation of the $\mathrm{CW}$ (8-10). The performance of bilateral ASCP according to the anatomic information on cerebral and carotid arteries will maintain sufficient cerebral perfusion during aortic arch surgery, and thus may be useful to reduce postoperative neurologic complications (11). However, except for CTA, the preoperative assessments of the variations of $\mathrm{CW}$ using other imaging modalities such as transcranial Doppler ultrasound (TCD) or magnetic resonance angiography (MRA) are impractical in emergency situations like ATAAD (6).

Additionally, detailed information on the involvement of carotid vessels is essential for surgical procedures. The hypoplasia or occlusion of right internal carotid artery (ICA) or VA may also lead to latent hypoperfusion of the left cerebral hemisphere during unilateral ASCP. These should be payed close attention by the surgeon. Hence, the identification of these carotid pathology and anatomy is critical for surgeons to plan the details of operative procedures prior to complex aortic arch surgery.

The advances in latest CT technology have made single imaging of a long scan range possible $(12,13)$. Therefore, combined CTA imaging of head, neck and aorta using dual source CT (DSCT) may also be feasible. According to the best of our knowledge, no previous studies have reported the comprehensive evaluation of craniocervical arteries and aorta in patients with ATAAD.

The purpose of this study was to evaluate the image quality and radiation dose of a comprehensive CTA protocol that expands the conventional aortic CTA coverage to head and neck. Specifically, we attempt to explore whether the new aortic CTA protocol benefit the surgeon by providing useful information of craniocervical pathology and anatomy that aids to plan appropriate ASCP method and surgical procedures in patients with ATAAD.

\section{Methods}

\section{Patients}

This prospective observational study was conducted in the largest hospital of northwest China. This prospective study was approved by our institutional review board and all patients provided written informed consent. From October 2013 to March 2016, a total of 1,125 patients with clinically suspected aortic dissection received the new aortic CTA protocol. ATAAD was defined as any nontraumatic dissection of the aorta proximal to the left subclavian artery presenting within 14 days of symptom onset (14). Exclusion criteria included: (I) non-dissection aortic diseases or normal aorta ( $\mathrm{n}=78)$; (II) type $\mathrm{B}$ aortic dissection ( $\mathrm{n}=667)$; (III) postoperation of aortic dissection ( $\mathrm{n}=107)$; (IV) chronic type A aortic dissection ( $\mathrm{n}=16)$; (V) renal insufficiency (serum creatinine level greater than $1.5 \mathrm{mg} / \mathrm{dL}, \mathrm{n}=12$ ); (VI) history of hypersensitivity to iodinated contrast medium $(n=2)$. Finally, 243 patients with ATAAD were enrolled in the present analysis.

\section{CT data acquisition and postprocessing}

All examinations were performed using a second-generation DSCT (Somatom Definition Flash, Siemens Healthcare, Forchheim, Germany) with high-pitch spiral scan mode. Patients received a combined CTA imaging of head, neck and aorta in a cranio-caudal direction from the top of the calvaria to the pubic symphysis. The scanning parameters were as follows: a tube voltage of $100 \mathrm{kV}$, pitch of 3.0 , slice collimation of $2 \mathrm{~mm} \times 128 \mathrm{~mm} \times 0.6 \mathrm{~mm}$ by means of a z-flying focal spot, gantry rotation time of $280 \mathrm{~ms}$, attenuation-based tube current modulation with a reference tube current time product set at $300 \mathrm{mAs}$ per rotation. For all scans, patients were positioned supine with both arms up.

Each patient received an injection of $70 \mathrm{~mL}$ of iopromide (Ultravist 370, $370 \mathrm{mg} \mathrm{I} / \mathrm{mL}$, Bayer Schering Pharma, Berlin, Germany) at a flow rate of $5 \mathrm{~mL} / \mathrm{s}$ followed by $40 \mathrm{~mL}$ saline solution. Bolus tracking was performed in the suprarenal descending aorta with an attenuation threshold of $100 \mathrm{HU}$.

Images were reconstructed with a slice thickness of $1 \mathrm{~mm}$ and an increment of $0.8 \mathrm{~mm}$. A smooth tissue convolution 
kernel (B26f) was applied. All images were analyzed by two experienced cardiovascular radiologists.

\section{Measurement of radiation dose}

The volume CT dose index (CTDIvol) and dose-length product (DLP) were obtained for all scans using the dose exposure record generated by the scanner console. The DLP can be related to energy imparted to organs and can thus be used to assess overall radiation burden of a given examination. It is equal to the product of the CTDIvol multiplied by the length of the scan in centimeters (15).

\section{Image quality of craniocervical arteries}

The images of all cerebral and carotid arteries were analyzed and graded in consensus by two cardiovascular radiologists. The arteries of per dataset were divided into 19 segments for analysis. These vessel segments include anterior cerebral artery (ACA), middle cerebral artery (MCA), posterior cerebral artery (PCA), anterior communicating artery (AcomA), posterior communicating artery (PcomA), basilar artery (BA), ICA, CCA, VA, innominate artery (IA), and subclavian artery (SA). For each arterial segment, image quality was graded on a 3 -point scale ( $0=$ excellent, $1=$ good, and $2=$ nondiagnostic $)(12)$. The percentage of diagnostic images was calculated by dividing the number of good or excellent vessels by the total number of assessed vessel segments.

\section{CTA findings of carotid arteries}

Dissection involving supraaortic vessels was identified. The extension of intimal flap into uni- or bilateral CCA and stenosis $>50 \%$ of the true lumen were described. Hypoplasia of carotid arteries was defined as the diameters less than $2.2 \mathrm{~mm}$ (16). The tortuosity of carotid artery was defined on the basis of previous report (17). Hypodensity of carotid arteries in one side was also described. The number and rate of each involved artery and abnormal vessel were recorded.

\section{CTA findings of cerebral arteries and the variations in the $C W$}

Each component stems of the $\mathrm{CW}$ as well as the intracranial VA were carefully analyzed and measured on the best slice of the CT axial images. Hypoplasia of cerebral arterial or intracranial VA was defined as a diameter less than $1 \mathrm{~mm}$ $(10,11)$. Both hypoplasia and absence were considered as haemodynamic significance. Previously work (18) has demonstrated that insufficient cross-perfusion mainly in cases with multiple abnormalities within the CW. For the patients underwent aortic arch surgery, we listed all types encompassed at least two variable component stems that could lead to hypoperfusion in theory during unilateral ASCP. Other CTA findings were also described.

\section{Statistical analysis}

Statistical analysis was performed on software SPSS (Version 19.0, SPSS Inc., Chicago IL, USA). Categorical variables were presented as frequency values and proportions. Continuous normally distributed variables were presented as mean \pm standard deviation (SD). Cohen's Kappa statistics were calculated for inter-reader agreements for the assessment of image quality. A kappa value of greater than 0.81 was considered as excellent, values of $0.61-0.80$ as good, values of $0.41-0.60$ as moderate and values below 0.20 as poor agreement.

\section{Results}

\section{Patient population}

All enrolled patients underwent successful single CTA scanning of head, neck and aorta on DSCT. Demographics and clinical features are displayed in the Table 1 .

\section{Radiation dose}

The mean CTDIvol and DLP of our new CTA protocol were $3.36 \pm 0.25 \mathrm{mGy}$ and $314.11 \pm 29.31 \mathrm{mGy} . \mathrm{cm}$, respectively.

\section{Image quality of craniocervical arteries}

A total of 4,272 craniocervical vessel segments were evaluated (Table 2). The average image quality score of each carotid artery segment was $<1$. Except AcomA (1.58), the average image quality scores of other cerebral artery segments were all $<1.5$. The cerebral and carotid arteries were $100 \%$ diagnostic (rated good or excellent) in all study patients. Interobserver agreement was excellent for all craniocervical vessel segments (kappa coefficients) $>0.81$.

\section{CTA findings of carotid arteries}

The CTA findings of carotid arteries are recorded in the Table 3. Approximately 47\% (115/243) of patients presented dissection involving carotid arteries. The involved carotid 
Table 1 Patient characteristics

\begin{tabular}{lc}
\hline Characteristics & $\mathrm{n}(\%)(\mathrm{N}=243)$ \\
\hline Age (years) & $52.6 \pm 14.6$ \\
Male & $207(85.2)$ \\
Body mass index $\left(\mathrm{kg} / \mathrm{m}^{2}\right)$ & $24.4 \pm 3.1$ \\
Hypertension & $209(86.0)$ \\
Coronary artery disease & $10(4.1)$ \\
Previous stroke & $16(6.6)$ \\
Marfan syndrome & $14(5.8)$ \\
Aortic aneurysm & $19(7.8)$ \\
Diagnostic imaging & \\
Chest radiograph & $29(11.9)$ \\
Computed tomographic angiography & $215(88.5)$ \\
Transthoracic echocardiogram (TTE) & $176(72.4)$ \\
Carotid Doppler ultrasonography & $8(3.3)$ \\
Abdominal Doppler ultrasonography & $12(4.9)$ \\
Magnetic resonance imaging & $30(12.3)$ \\
\hline
\end{tabular}

Values are expressed as mean \pm SD or $n(\%)$. SD, standard deviation. arteries were most common in the IA $(21.0 \%)$ followed by CCA $(19.8 \%)$, SA (13.6\%) and ICA (2.9\%). Twenty-nine CCAs $(29 / 48,60.4 \%)$ with stenosis $>50 \%$ in true lumen (Figure 1) was detected in a total of 48 involved CCAs. Dissection involving whole or proximal CCA was observed in $37.5 \%(18 / 48)$ and $27.1 \%(13 / 48)$ of involved CCAs, respectively. Hypoplasia (Figure 2A), occlusion (Figure 2B), and the variation of the VA (Figure $2 C$ ) were also showed and recorded in Table 2. The tortuous of carotid arteries, including ICA, VA and CCA tortuosity (Figure 2D-F), was the most frequent findings and was found in $90.9 \%$ of patients. In addition, hypodensity (Figure $2 G$ ) of carotid arteries was found in 19 patients owing to the intimal flap involving in or vessels originating from false lumen.

\section{CTA findings of cerebral arteries and hemodynamic variation types of the $C W$}

In 243 patients, 209 (86\%) had variation of CW. One, two or three hypoplastic/absent component stems were detected in $41(16.9 \%), 83$ (34.2\%), and 85 (35\%) patients, respectively. Seven hemodynamic variation types of the CW (Figure 3) were detected in 68 patients $(28.0 \%)$ in the setting of hypothesized

Table 2 Image quality of cerebral and carotid arteries $(\mathrm{N}=243)$

\begin{tabular}{|c|c|c|c|}
\hline Arterial segment & Numbers & Mean score & Diagnostic segments \\
\hline Anterior cerebral artery & 486 & $1.37 \pm 0.38$ & $486(100 \%)$ \\
\hline Middle cerebral artery & 486 & $1.15 \pm 0.44$ & $486(100 \%)$ \\
\hline Posterior cerebral artery & 482 & $1.23 \pm 0.35$ & $482(100 \%)$ \\
\hline Posterior communicating artery & 215 & $1.37 \pm 0.32$ & $215(100 \%)$ \\
\hline Basilar artery & 243 & $1.24 \pm 0.17$ & $243(100 \%)$ \\
\hline Carotid artery & 2,184 & & \\
\hline Internal carotid artery & 486 & $0.73 \pm 0.22$ & $486(100 \%)$ \\
\hline Innominate artery & 243 & $0.35 \pm 0.25$ & $243(100 \%)$ \\
\hline Subclavian artery & 486 & $0.58 \pm 0.24$ & $486(100 \%)$ \\
\hline Total & 4,272 & & $4,272(100 \%)$ \\
\hline
\end{tabular}

Values are expressed as mean \pm SD or $n(\%)$. SD, standard deviation. 
Table 3 CTA findings of carotid arteries (N=243)

\begin{tabular}{|c|c|c|c|c|}
\hline CTA findings & n (\%) (total) & n (\%) (left) & n (\%) (right) & n (\%) (bilateral) \\
\hline IA & $51(21.0)$ & & & \\
\hline CCA & $48(19.8)$ & $7(2.9)$ & $24(9.9)$ & $17(7.0)$ \\
\hline SA & 33 (13.6) & $13(5.3)$ & $15(6.2)$ & $5(2.1)$ \\
\hline Hypodensity & $19(7.8)$ & & & \\
\hline ICA & $5(2.1)$ & 0 & $5(2.1)$ & 0 \\
\hline $\mathrm{CCA}+\mathrm{ICA}$ & $10(4.1)$ & 0 & $10(4.1)$ & 0 \\
\hline VA & $4(1.6)$ & 0 & $4(1.6)$ & 0 \\
\hline VA & $24(9.9)$ & $10(4.1)$ & $14(5.8)$ & 0 \\
\hline Plaque & 30 (12.3) & & & \\
\hline ICA & $19(7.8)$ & $10(4.1)$ & $3(1.2)$ & $6(2.5)$ \\
\hline VA & $3(1.2)$ & $1(0.4)$ & $2(0.8)$ & 0 \\
\hline SA & $5(2.1)$ & $2(0.8)$ & $3(1.2)$ & 0 \\
\hline CCA & $3(1.2)$ & 0 & $2(0.8)$ & $1(0.4)$ \\
\hline Tortuosity & 221 (90.9) & & & \\
\hline ICA & $215(88.5)$ & $22(9.1)$ & $4(1.6)$ & 190 (78.2) \\
\hline
\end{tabular}

Values are expressed as $n(\%)$. CTA, computed tomography angiography; IA, innominate artery; CCA, common carotid artery; SA, subclavian artery; ICA, internal carotid artery; VA, vertebral artery.

unilateral ASCP. The number and rate of variations in seven types as well as vessel territories at risk of hypoperfusion during unilateral ASCP are presented in Table 4. Type I with hypo/ aplasia of AcomA and left PcomA was the most frequent variation type and was found in 16.5\% (40/243) of patients (Figure 3A). The remaining 6 types were uncommon and each type was found in less than $4 \%$ of patients (Figure 3B-7). Other intracranial CTA findings included arterial stenosis or occlusion $(8 / 243,3.3 \%)$, aneurysm (4/243, 1.6\%) and hypodensity (20/243, 8.2\%) of cerebral arteries.

\section{Comprebensive analysis of CTA findings}

Although the CTA findings of cerebral and carotid arteries were separately shown in different tables, the clinical analysis of craniocervical anatomy and pathology, as well as aortic pathology in each ATAAD case was comprehensive. The valuable information on aortic and craniocervical arteries that could influence the plan of surgical procedures and the choice of ASCP method was recorded and discussed with the surgeon preoperatively. 


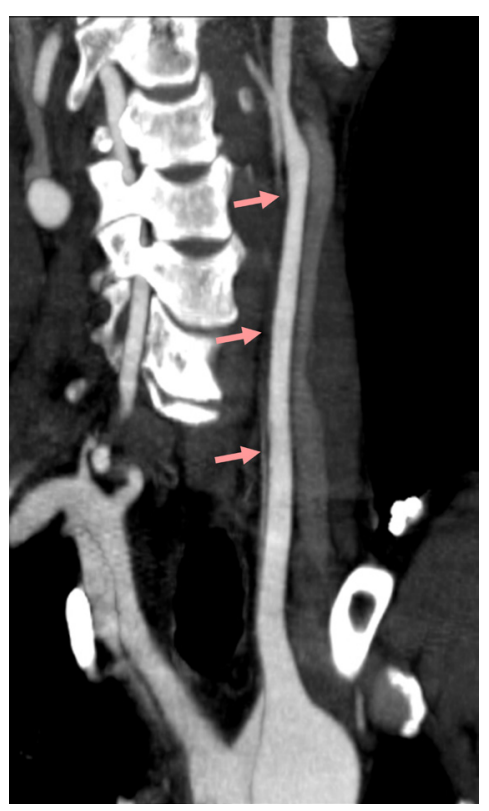

Figure 1 Coronal thin MIP shows aortic dissection involving whole left CCA and severe narrowing in the true lumen (arrow). MIP, maximum intensity projection; CCA, common carotid artery.

\section{Discussion}

Clinical presentation of acute aortic dissection is a true emergency, often with dramatic scenario, with patients in critical conditions. Due to the acute and complex nature of ATAAD, the use of multi-detector CT has been shown to be an invaluable tool in the emergency department (19).

Furtado et al. (12) demonstrated the feasibility of a combined imaging of the brain, carotid arteries, aorta, and heart by using a 64-section CT scanner. Their new CT protocol achieved excellent image quality in ischemic stroke patients. Another study (13) also reported a single evaluation of heart, head, and neck CTA using a DSCT scanner. The new CT protocol provided good image quality of coronary, cerebral and carotid arteries at a low radiation dose. Our results, showing 100\% diagnostic image quality of aorta and craniocervical arteries, further demonstrated the feasibility of imaging a long body range with this new aortic CTA protocol.

Due to added scan length of head and neck, higher radiation dose is possible using our new aortic CTA protocol. Fujioka et al. (20) conducted a systematic analysis of dose reduction in 30 patients undergoing 64-MDCT angiography of the aorta and coronary arteries. Their CTA protocol, with a coverage from pulmonary apex to the pelvis, a tube voltage of $100 \mathrm{kV}$ and a tube current of $600 \mathrm{mAs}$, delivered a mean DLP of $444 \pm 98$ mGy.cm. Mileto et al. (21) performed a conventional aortic CTA using highpitch DSCT, with the setting of $120 \mathrm{kV}$ and $200 \mathrm{mAs}$. The mean CTDIvol was $8.3 \pm 0.7 \mathrm{mGy}$. Our new aortic CTA protocol, with the setting of $100 \mathrm{kV}, 300 \mathrm{mAs}$, and a high pitch of 3.0, however, delivered a mean CTDIvol of $3.36 \pm 0.25 \mathrm{mGy}$ and DLP of $314.11 \pm 29.31 \mathrm{mGy} . \mathrm{cm}$, which significantly was lower than these conventional aortic CTA studies with shorter scan range. Therefore, this new protocol has the potential to provide diagnostic image quality of aorta and craniocervical arteries at low radiation exposure.

An appropriate cerebral protection method is a key issue in aortic arch surgery with HCA. It has been shown that about $17 \%$ of patients were likely to have inadequate perfusion of the left hemisphere with unilateral ASCP because of an incomplete CW (22). In this setting, bilateral ASCP is essential for restoration of perfusion in the left hemisphere. These may lower the occurrence of postoperative TND $(5,7)$. Thus, the anatomy of the cerebral arteries of the patients undergoing the aortic arch surgery should be examined for better risk stratification. Our new aortic CTA protocol, with the capability of combined imaging craniocervical arteries and aorta in a very short time, better than other examinations that could not be underwent in emergency situations like ATAAD. Sixty-eight patients with 7 hemodynamic CW variation types in the case of unilateral ASCP were classified based on incremental cerebral CTA findings, which apprised the surgeon of the administration of bilateral ASCP. Of the 147 subjects who underwent aortic arch surgery, 31 (21.1\%) were administered with bilateral ASCP while unilateral ASCP was a conventional choice in our institution in the last few years.

CTA findings of carotid arteries in ATAAD patients are rarely described in previous reports due to the conventional scan coverage from proximal supraaortic branches to iliac arteries $(2,3,23)$. The details of carotid lesions such as the extension of dissection in CCA, reentry in CCA as well as thrombosis in false lumen of CCA, however, are vital for surgery procedures of ATAAD. Significant stenosis of true lumen in CCA, hypodensity in CCA and ICA, and hypoplasia/occlusion of right VA or ICA (8) all indicate the possibility of cerebral hypoperfusion. Therefore, our new aortic CTA imaging benefited the surgeon by visualizing of carotid pathologic and anatomy that could aid the optimal preoperative planning and intraoperative processing. 

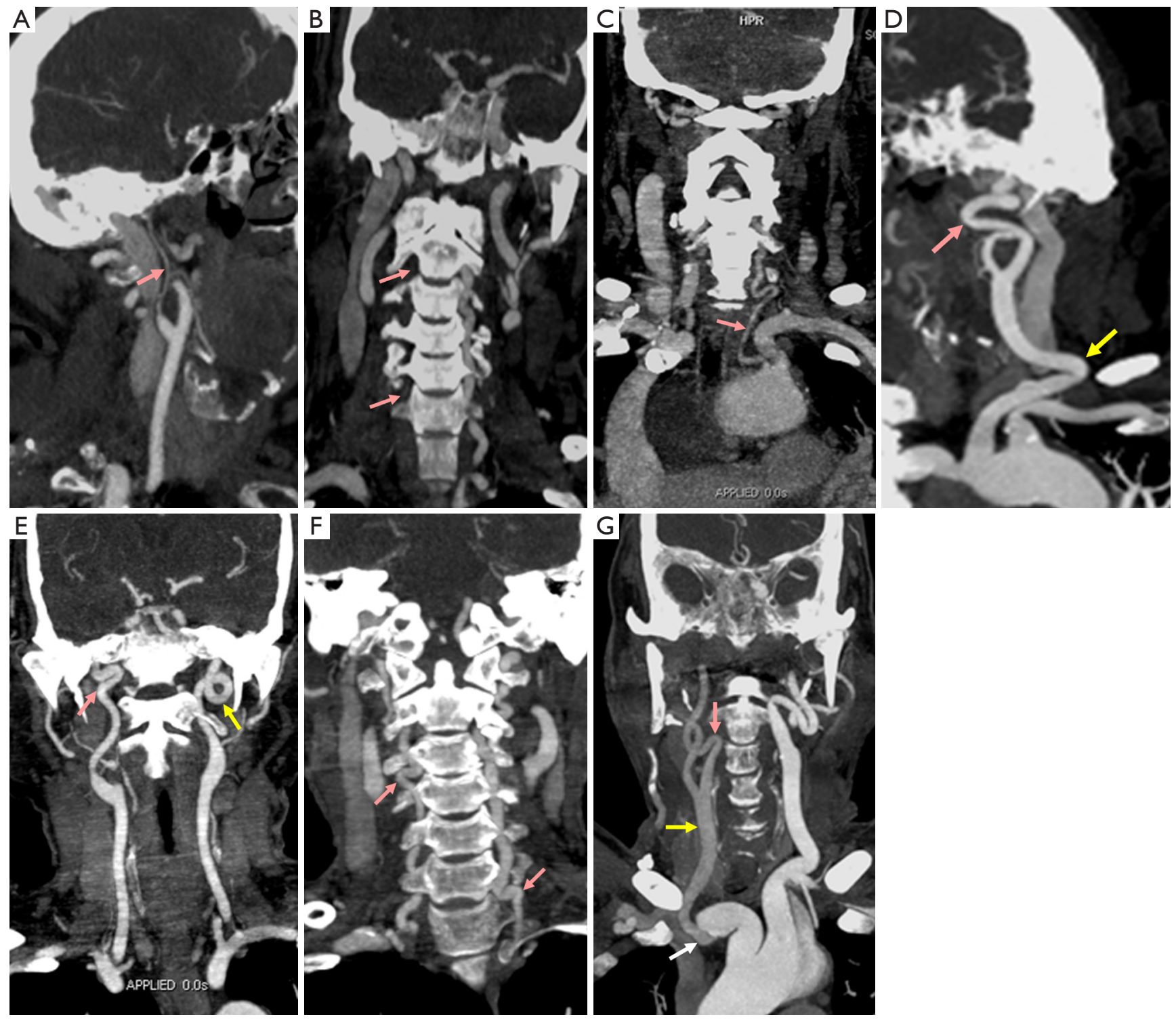

Figure 2 Incremental CTA findings of carotid arteries. (A) Sagittal thin MIP image shows hypoplasia of right ICA (arrow); (B) coronary thin MIP image shows occlusion of right VA (arrow); (C) coronary thin MIP image shows tortuous left VA abnormally originating from aortic arch (arrow); (D) coronary thin MIP image shows the kinking of left ICA (pink arrow) and CCA (yellow arrow); (E) coronary thin MIP image shows the kinking of right ICA (pink arrow) and coiling of left ICA (yellow arrow); (F) coronary thin MIP image shows the kinking of bilateral VAs (arrow); (G) coronary thin MIP image shows hypodensity of right ICA (pink arrow) and CCA (yellow arrow) due to the right CCA originating from the false lumen (white arrow). CTA, computed tomography angiography; MIP, maximum intensity projection; ICA, internal carotid artery; VA, vertebral artery; CCA, common carotid artery.

It is perhaps noteworthy that a dramatically high frequency $(90.9 \%)$ of carotid artery tortuosity was found in our cohort. Previous results have demonstrated that tortuous carotid artery is often detected in hypertensive patients (24-26). Several reports $(27,28)$ also indicated that arterial tortuosity of the head and neck vessels is present in $84 \%$ to $100 \%$ of patients with Loeys-Dietz syndrome. However, there are no previous reports on the phenomenon of tortuous carotid artery in patients with ATAAD. Our study demonstrates that carotid arterial tortuosity is also common 

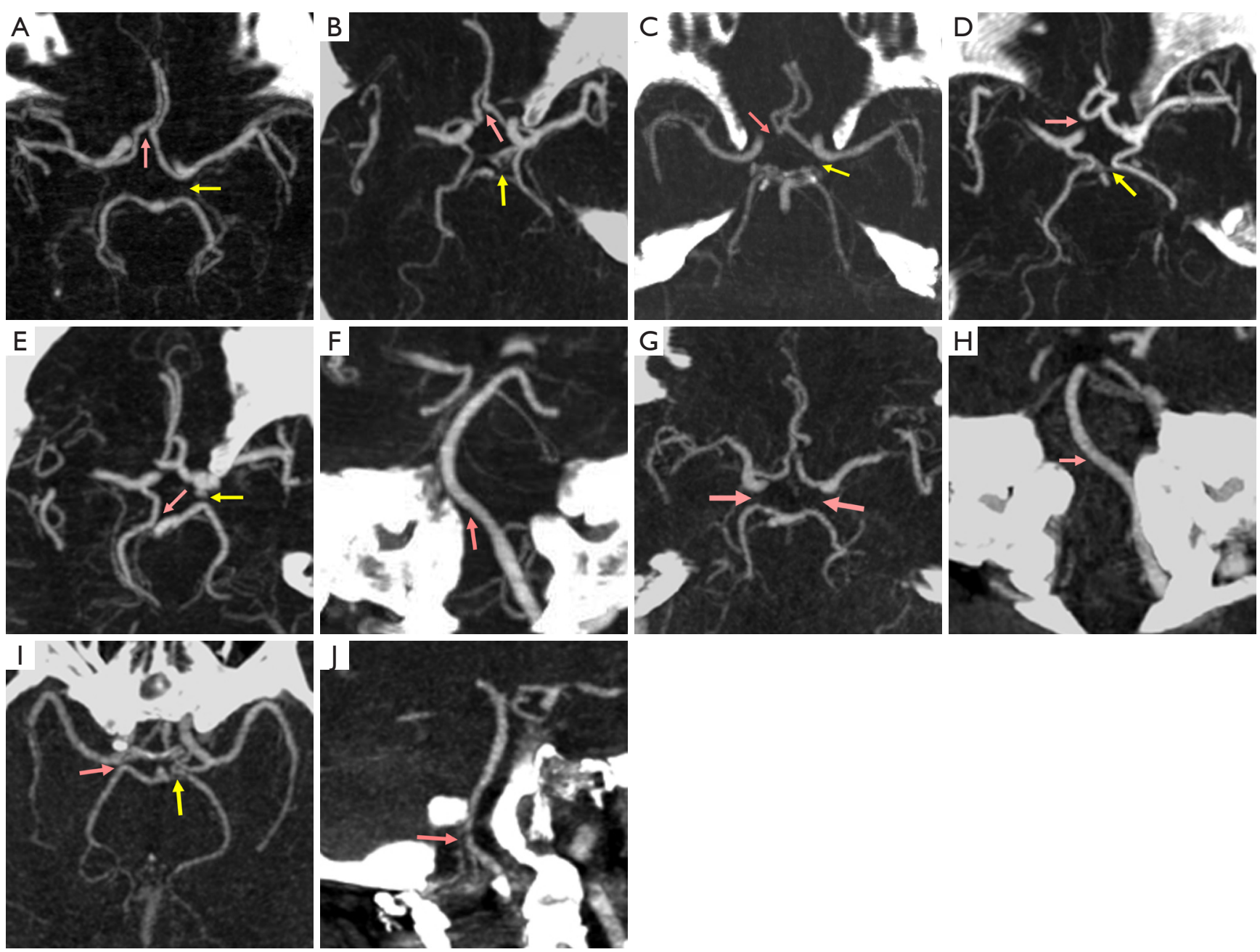

Figure 3 CTA classification of 7 hemodynamic CW variation types during unilateral ASCP. (A) Variation type I with absence of AcomA (pink arrow) and left PcomA (yellow arrow); (B) type II with absence of AcomA (pink arrow) and left P1 (yellow arrow); (C) type III with absence of right A1 (pink arrow) and left PcomA (yellow arrow); (D) type IV with absence of right A1 (pink arrow) and left P1 (yellow arrow). Type $\mathrm{V}$ with absence of right P1 (pink arrow), left PcomA (yellow arrow) (E); and intracranial right VA (pink arrow) (F). Type VI with absence of bilateral PcomA (pink arrow) (G) and intracranial right VA (pink arrow) (H). Type VII with absence of right PcomA (pink arrow), left P1 (yellow arrow) (I); and hypoplasia of right VA (pink arrow) (J). CTA, computed tomography angiography; CW, the circle of Willis; ASCP, antegrade selective cerebral perfusion; AcomA, anterior communicating artery; PcomA, posterior communicating artery; P1, precommunicating segment of posterior cerebral artery; A1, precommunicating segment of anterior cerebral artery; VA, vertebral artery.

in patients with ATAAD. It is well known that the majority of patients with aortic dissection have a history of hypertension. Approximately $86 \%$ of patients have hypertension in our cohort. The presentation of tortuous carotid artery in ATAAD is thereby likely to be associated with hypertension. Future studies should to be carried out to elucidate the underlying mechanism of tortuous carotid artery in ATAAD.

There were several limitations to our study. First, this study was a prospective analysis and thus, is subject to all of the potential limitations intrinsic to this type of study design. Our study was performed at a single urban, academic site so the number of patients and the skills of imaging and surgery reflect our specific institutional idiosyncrasies. Additionally, we didn't compare our new protocol with conventional aortic CTA protocol for effect on clinical outcomes because surgeons prefer the new protocol to the standard aortic CTA protocol. Thus, future randomized trials should be performed to determine the potential benefit of the 
Table 4 CTA classification of 7 hemodynamic CW variation types in the setting of unilateral ASCP (N=68)

\begin{tabular}{llcl}
\hline Classification & Variation of component Stems & Number (\%) & Latent hypoperfusion in vessel territories during unilateral ASCP \\
\hline Type I & AcomA + LPcomA & $40(16.5)$ & $\begin{array}{l}\text { Left anterior cerebral artery + left middle cerebral artery } \\
\text { Left anterior cerebral artery + left middle cerebral artery + left } \\
\text { posterior cerebral artery }\end{array}$ \\
Type III & AcomA + LP1 & $2(0.8)$ & Bilateral anterior cerebral artery + left middle cerebral artery \\
Type IV & RA1 + LPcomA & $8(3.3)$ & Bilateral anterior cerebral artery + left middle cerebral artery + left \\
pype & $4(1.6)$ & $2(0.8)$ & Left posterior cerebral artery \\
Type VI & RVA + RP1 + LPcomA & $8(3.3)$ & Bilateral posterior cerebral artery \\
Type VII & RVA + RPcomA + LP1 & $4(1.6)$ & Right posterior cerebral artery \\
Total & $68(28.0)$ & \\
\hline
\end{tabular}

Values are expressed as $\mathrm{n}(\%)$. CTA, computed tomography angiography; CW, the circle of Willis; ASCP, antegrade selective cerebral perfusion; AcomA, anterior communicating artery; PcomA, posterior communicating artery; A1, precommunicating segment of anterior cerebral artery; P1, precommunicating segment of posterior cerebral artery; VA, vertebral artery.

incremental CTA findings in therapeutic decision making to improved clinical outcomes in patients with ATAAD.

In conclusion, this study demonstrates that a combined CTA of head, neck and aorta in patients with ATAAD is feasible, with low radiation dose and diagnostic image quality. Incremental craniocervical information benefit the surgeon by providing useful cerebral and carotid pathology and anatomy that aids to plan detailed surgical procedures and appropriate cerebral protection method in patients with ATAAD.

\section{Acknowledgements}

None.

\section{Footnote}

Conflicts of Interest: The authors have no conflicts of interest to declare.

Ethical Statement: The study was approved by our institutional review board and written informed consent was obtained from all patients.

\section{References}

1. Salvolini L, Renda P, Fiore D, et al. Acute aortic syndromes: role of multi-detector row CT. Eur J Radiol 2008;65:350-8.

2. Hiratzka LF, Bakris GL, Beckman JA, et al. 2010 ACCF/

\section{AHA/AATS/ACR/ASA/SCA/SCAI/SIR/STS/ SVM} guidelines for the diagnosis and management of patients with thoracic aortic disease: executive Summary. Catheter Cardiovasc Interv 2010;76:E43-86.

3. Yoo SM, Lee HY, White CS. MDCT evaluation of acute aortic syndrome. Radiol Clin North Am 2010;48:67-83.

4. Morimoto N, Okada K, Okita Y. Lack of neurologic improvement after aortic repair for acute type A aortic dissection complicated by cerebral malperfusion: predictors and association with survival. J Thorac Cardiovasc Surg 2011;142:1540-4.

5. Hagl C, Ergin MA, Galla JD, et al. Neurologic outcome after ascending aorta-aortic arch operations: effect of brain protection technique in high-risk patients. J Thorac Cardiovasc Surg 2001;121:1107-21.

6. Kazui T. Which is more appropriate as a cerebral protection method-unilateral or bilateral perfusion? Eur J Cardiothorac Surg 2006;29:1039-40.

7. Di Eusanio M, Schepens MA, Morshuis WJ, et al. Brain protection using antegrade selective cerebral perfusion: a multicenter study. Ann Thorac Surg 2003;76:1181-88; discussion 1188-89.

8. Papantchev V, Hristov S, Todorova D, et al. Some variations of the circle of Willis, important for cerebral protection in aortic surgery-a study in Eastern Europeans. Eur J Cardiothorac Surg 2007;31:982-9.

9. De Silva KR, Silva R, Amaratunga D, et al. Types of the cerebral arterial circle (circle of Willis) in a Sri Lankan Population. BMC Neurol 2011;11:5. 
10. Ansari S, Dadmehr M, Eftekhar B, et al. A simple technique for morphological measurement of cerebral arterial circle variations using public domain software (Osiris). Anat Cell Biol 2011;44:324-30.

11. Papantchev V, Stoinova V, Aleksandrov A, et al. The role of Willis circle variations during unilateral selective cerebral perfusion: a study of 500 circles. Eur J Cardiothorac Surg 2013;44:743-53.

12. Furtado AD, Adraktas DD, Brasic N, et al. The triple rule-out for acute ischemic stroke: imaging the brain, carotid arteries, aorta, and heart. AJNR Am J Neuroradiol 2010;31:1290-6.

13. Wang Z, Chen Y, Wang Y, et al. Feasibility of low-dose contrast medium high pitch CT angiography for the combined evaluation of coronary, head and neck arteries. PloS One 2014;9:e90268.

14. Bossone E, Corteville DC, Harris KM, et al. Stroke and outcomes in patients with acute stroke and outcomes in patients with acute type A aortic dissection. Circulation 2013;128:S175-9.

15. Hamberg LM, Rhea JT, Hunter GJ, et al. Multi-detector row CT: radiation dose characteristics. Radiology 2003;226:762-72.

16. Sano A, Hirano T, Watanabe K, et al. Preoperative evaluation of the vertebral arteries and posterior portion of the circle of Willis for cervical spine surgery using 3-dimensional computed tomography angiography. Spine 2013;38:E960-67.

17. Han HC. Twisted blood vessels: symptoms, etiology and biomechanical mechanisms. J Vasc Res 2012;49:185-97.

18. Hoksbergen AW, Legemate DA, Ubbink DT, et al. Collateral variations in circle of Willis in atherosclerotic population assessed by means of transcranial color-coded duplex ultrasonography. Stroke 2000;31:1656-60.

19. Moore AG, Eagle KA, Bruckman D, et al. Choice of computed tomography, transesophageal echocardiography, magnetic resonance imaging and aortography in acute

Cite this article as: Wen D, Zhao H, Duan W, An R, Li J, Zheng M. Combined CT angiography of the aorta and craniocervical artery: a new imaging protocol for assessment of acute type A aortic dissection. J Thorac Dis 2017;9(11):47334742. doi: $10.21037 / j t d .2017 .09 .28$ aortic dissection: International Registry of Acute Aortic Dissection (IRAD). Am J Cardiol 2002;89:1235-8.

20. Fujioka C, Horiguchi J, Kiguchi M, et al. Survey of aorta and coronary arteries with prospective ECG-triggered 100-kV 64-MDCT angiography. AJR Am J Roentgenol 2009;193:227-33.

21. Mileto A, Ramirez-Giraldo JC, Nelson RC, et al. High-pitch dual-source MDCT for imaging of the thoracoabdominal aorta: relationships among radiation dose, noise, pitch, and body size in a phantom experiment and clinical study. AJR Am J Roentgenol 2015;205:834-9.

22. Merkkola P, Tulla H, Ronkainen A, et al. Incomplete circle of Willis and right axillary artery perfusion. Ann Thorac Surg 2006;82:74-9.

23. Beeres M, Schell B, Mastragelopoulos A, et al. High-pitch dual-source CT angiography of the whole aorta without ECG synchronisation: initial experience. Eur Radiol 2012;22:129-37.

24. Del Corso L, Moruzzo D, Conte B, et al. Tortuosity, kiking, and coiling of the carotid artery: expression of atherosclerosis or aging? Angiology 1998;49:361-71.

25. Pancera P, Ribul M, presciuttini B, et al. Prevalence of carotid artery kiking in 590 consecutive subjects evaluated by echo-color Doppler. Is there a correlation with arterial hypertension? J Intern Med 2000;248:7-12.

26. Oliviero U, Scherillo G, Casaburi C, et al. Prospective evaluation of hypertensive patients with carotid kiking and coiling: an ultrasonographic 7-year study. Angiology 2003;54:169-75.

27. Loeys BL, Chen J, Neptune ER, et al. A syndrome of altered cardiovascular, craniofacial, neurocognitive and skeletal development caused by mutations in TGFBR1 or TGFBR2. Nat Genet 2005;37:275-81.

28. Rodrigues VJ, Elsayed S, Loeys BL, et al. Neuroradiologic manifestations of Loeys-Dietz syndrome type 1. AJNR Am J Neuroradiol 2009;30:1614-9. 